\title{
Estados Unidos y China tensiones por el liderazgo de la región Pacífico Occidental
} The United States and China: Tensions for the leadership of Western Pacific Ocean

Sol Revelo y David Revelo* 


\section{Resumen}

En los últimos años, la región Pacífico Occidental ha sido el foco de grandes tensiones entre Estados de la zona. Estas tensiones han ocurrido principalmente por las disputas territoriales en el Mar del Sur de China. En específico, las pretensiones territoriales de la República Popular China para liderar en el Mar del Sur de China han desafiado a la primicia y liderazgo de los Estados Unidos en la región Pacífico Occidental. En este artículo se analizan las dinámicas de poder en la región Pacífico Occidental y las estrategias que la República Popular China ha usado para liderar esta zona.

\section{Palabras clave:}

República Popular China, Estados Unidos, dinámicas de poder, influencia, estrategia, Mar del Sur de China, CONVEMAR. 


\section{Ahstract}

In recent years, the Western Pacific region has been the field of great tensions between states in the area. These tensions have mainly occurred due to territorial disputes in the South China Sea. Specifically, the territorial claims of the People's Republic of China to lead in the South China Sea have challenged the leadership of the United States in the Western Pacific region. This article analyzes the power dynamics in the Western Pacific region and the strategies that the People's Republic of China has used to lead this area.

\section{Keywords:}

People's Republic of China, United States, power dynamics, influence, strategy, South China Sea, UNCLOS. 
¿Está el Sistema Internacional presenciando un giro en las dinámicas de poder en la zona del Pacífico Occidental? Con el fin de la Guerra Fría, Estados Unidos (EE. UU.) pudo consolidar su alcance e influencia dentro de la zona Pacífico Occidental, a través de una histórica construcción de relaciones políticas y económicas con Estados como Japón, Filipinas, la República de Corea, Australia, Vietnam, Indonesia, Malasia y, cautelosamente, Taiwán (The White House 2017, 45-47). Las diferentes administraciones estadounidenses han enfatizado la importancia de mantener un papel activo dentro de la zona Pacífico Occidental. En efecto, en la última publicación de la Estrategia de Seguridad Nacional de Estados Unidos se enfatiza la importancia de mantener el respeto del Derecho Internacional, el libre mercado, y la defensa y seguridad de los Estados de la región a través de la expansión de relaciones políticas, económicas y militares (The White House 2017, 45-47).

Esta dinámica de poder ha encontrado un creciente reto propuesto por la República Popular China (RPC), que ha trabajado para establecerse como una potencia emergente durante los últimos 30 años. A través de diferentes hechos que han sucedido en la zona Pacífico Occidental durante los últimos años, es plausible decir que existe un intento por parte de la RPC de aumentar su influencia dentro de la zona. ¿Lo está logrando? En este artículo analizaremos cómo la creciente influencia regional de la República Popular China pretende desafiar las dinámicas de poder en la región Pacífico Occidental que, históricamente, han sido manejadas por Estados Unidos. Para tal análisis, nos enfocaremos en hechos recientes que han ocurrido en la zona Pacífica Occidental, ejemplificando la presión de la RPC a Taiwán. Un controversial actor del Sistema Internacional que mantiene acuerdos políticos con EE. UU. similares a los de otros Estados de la región. En segundo lugar, expondremos brevemente un importante instrumento de Derecho Internacional para la situación de la zona: La Convención sobre los Derechos del Mar. Posteriormente, pasaremos a examinar la situación en la región Pacífica Occidental, el papel de EE. UU. y los intereses de RPC en relación con sus acciones. Finalmente, concluimos con nuestro análisis sobre la situación actual en la región. 


\section{Contexto histórico}

El fin de la Segunda Guerra Mundial tuvo un impacto global que, efectivamente, lo vivimos hasta ahora. En específico, uno de los sucesos que impactaron a las dinámicas de poder en la región Pacífico Occidental fue la derrota de Japón ante las potencias aliadas de EE. UU., Reino Unido y la llamada República de China con la Conferencia del Cairo de 1943. Con este motivo, se expulsó a Japón de todas las islas en el Océano Pacífico que había ocupado desde la Primera Guerra Mundial y se devolvió a la República de China los territorios tomados incluyendo Manchuria, Taiwán, Isla de los Pescadores, entre otros (Department of State 2019). Sin embargo, el papel de la República de China cambió rápidamente dentro del Sistema Internacional. Durante la Segunda Guerra Mundial, el Partido Comunista de China ganó fuerza y apoyo dentro de la República de China. Esto desembocó en una escalada guerra civil entre 1947 y 1949 entre los dos, que provocó el traslado de la sede de gobierno de la República de China a la isla de Taiwán, y que el Partido Comunista de China, liderado por Mao Zedong, fundara la República Popular de China (RPC) en octubre de 1949 (Department of State 2019).

Después del fin de la Segunda Guerra Mundial, el sentimiento anticomunista y la estrategia de contención guiaron la política exterior de EE. UU. en su lucha contra la expansión comunista en general, principalmente promulgada por la Unión de Repúblicas Socialistas Soviéticas (URSS) (Hook y Spanier 2016, 34-36). Desde que la República de China desplazó su sede de gobierno a Taiwán en 1949, EE. UU. mantuvo su reconocimiento oficial y apoyo hacia la isla, con el fin de deslegitimar el gobierno comunista que estableció la RPC (Council on Foreign Relations 2019). La URSS había apoyado desde su inicio a la RPC, principalmente por su afiliación ideológica.

Sin embargo, desde 1960 las relaciones políticas entre la URSS y la RPC decayeron fuertemente por diferencias en sus enfoques de seguridad, culminando en 1969 en un conflicto fronterizo entre los dos Estados (Council on Foreign Relations 2019). Por la importancia política y militar de la RPC dentro del sistema internacional en aquel entonces, EE. UU. aceptó las invitaciones de acercamiento por parte de la RPC y, en 1979, 
EE. UU. finalmente reconoció a la RPC como un estado soberano, con su política de "Una Sola China", lo cual debilitó políticamente a la URSS (Council on Foreign Relations 2019). Por este mismo hecho, en 1979 EE. UU. se vio obligado a cortar relaciones diplomáticas oficiales con Taiwán, al reconocer a la RPC como la única China. Sin reconocer a Taiwán oficialmente, sin embargo, EE. UU. estableció rápidamente estrechas relaciones políticas con el gobierno de Taiwán, quien ha sido un aliado político estratégico en la región Pacífico Occidental. De estas relaciones hablaremos a continuación.

\section{Relación EE. UU. y la región Pacífica Occidental}

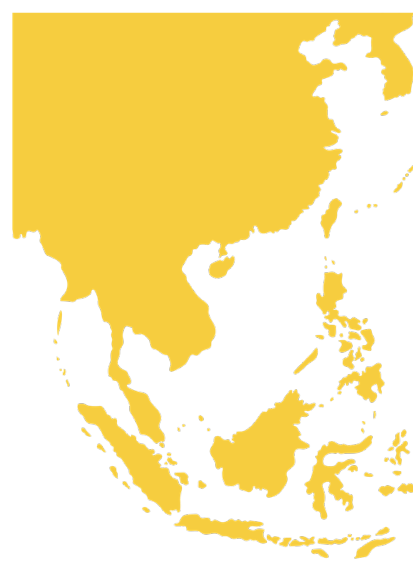

EE. UU. tiene alianzas formales y políticas en la región Pacífica Occidental, incluyendo a Taiwán, Japón, Filipinas, Corea del Sur y Tailandia (Congressional Research Service 2018). Al ser aliados por tratado, existe un acuerdo legalmente vinculante entre EE. UU. y cada uno de los Estados. Entre las características principa-

les de estos acuerdos, se recalca la dependencia de la presencia de EE. UU. para la estabilidad de la región del Pacífico Occidental, la asistencia militar necesaria para contrarrestar el ataque de cualquier enemigo, la importancia de mantener vínculos comerciales para el florecimiento económico, entre otros.

\subsection{Acta de Relaciones con Taiwán}

Como se mencionó previamente, aunque EE. UU. cortó relaciones diplomáticas oficiales con Taiwán en 1979, esto no significó un alejamiento político entre los dos. En efecto, EE. UU. estableció El Acta de Relaciones firmado por EE. UU. y Taiwán en 1979, el cual es un claro ejemplo de cómo EE. UU. reforzó su influencia y presencia con sus aliados tanto formales como políticos dentro de la región Pacífico Occidental. En el acuerdo, EE. 
UU. se compromete a contribuir en el sustento de la estabilidad de la región Pacífico Occidental y fortalecer relaciones comerciales entre los dos países (American Institute in Taiwan 2019). Además, en el acuerdo, EE. UU. se compromete a condenar cualquier tipo de actor, acción, o movimiento político que pueda poner en peligro el mantenimiento de la paz y seguridad de la región Pacífico Occidental (American Institute in Taiwan 2019). En términos más específicos, EE. UU. también se compromete en el acuerdo a proveer artículos y servicios de defensa a Taiwán, con el fin de que la Isla pueda ejercitar su propia defensa territorial ante alguna amenaza externa (American Institute in Taiwan 2019).

\subsection{Convención Sobre el Derecho del Mar}

En 1982 se firmó la Convención sobre el Derecho del Mar de las Naciones Unidas (CONVEMAR), un importante instrumento del Sistema Internacional. La CONVEMAR se trata de un acuerdo entre Estados que determina el territorio de mar, espacio aéreo sobre dicho territorio marítimo y los subsuelos de este, que corresponden a cada nación (ONU 1982). La RPC ratificó directamente en 1996 la convención, por lo que el país se encuentra vinculado legalmente a los acuerdos. Uno de los puntos más importantes de la CONVEMAR radica en que establece la posesión de una zona económica exclusiva a cada Estado dentro del acuerdo, en la que los recursos naturales de las aguas supra yacentes, el suelo marítimo, y su subsuelo, serían manejados soberanamente por cada Estado (ONU 1982, 51-52). De igual manera, dentro de la CONVEMAR se establece que el límite de la zona económica exclusiva de cada Estado no podría ser mayor a 200 millas náuticas desde su costa (ONU 1982, 51-52)

En sí, los principios de la CONVEMAR tienen un alcance global. Estados Unidos, sin necesariamente ser parte de esta convención, ha enfatizado fuertemente la importancia del respeto a este instrumento del Derecho Internacional dentro de la región Pacífico Occidental, implícitamente dirigiendo su afirmación a la RPC. Sin embargo, como el Derecho Internacional no presenta ningún mecanismo de sanción a los países que no cumplen con él, este ha sido utilizado instrumentalmente por las potencias globales (Allison 2016, 1). Consecuentemente, la RPC ha hecho en los últimos años declaraciones que han puesto en 
duda su comprometimiento con la CONVEMAR. Esto lo analizaremos más adelante. Tanto la CONVEMAR, como el Acta de Relaciones con Taiwán ejemplifican cómo EE. UU. se ha acercado y afirmado su presencia e influencia en la región Pacífica Occidental.

\section{Situación en la región del Pacífico Occidental}

La región Pacífica Occidental está conformada por 37 Estados, entre estos Brunei, la RPC, Filipinas, Indonesia, Malasia, Taiwán y Vietnam. Estos 6 países encuentran específicamente localizados en el Mar del Sur de China. Al ser una potencia creciente que es, la RPC ha demostrado su autoridad sobre otros territorios dentro de esta área, violando sus zonas económicas exclusivas de 200 millas marítimas. Sobre este hecho, la RPC ha argumentado que, históricamente, el territorio chino ha controlado el Mar del Sur de China y, adicionalmente, ha trazado su propia línea de territorio marítimo que engloba el $86 \%$ de tal Mar, cómo se puede apreciar en la Figura 1 (Allison 2016, 1).

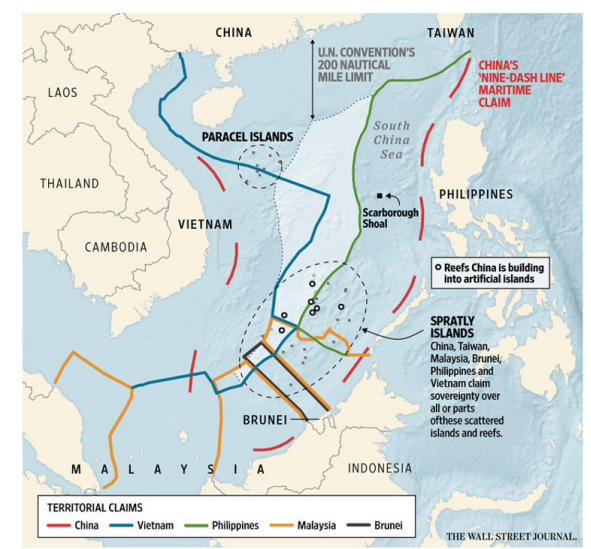

Figura 1: Pretensiones de la RPC en el Mar Sur de China (Black 2018)

En medio del Mar del Sur de China se encuentran las Islas Spratly, las cuales son ricas en recursos naturales como petróleo y gas, y además tienen una ubicación geográfica estratégica en la región Pacífica Occidental (Arana 2015). Al momento, estas islas se encuentran en medio de la disputa por diferentes territorios marítimos dentro del Mar del Sur de China. La RPC, por ejemplo, ha reclamado las Islas Spratly en su tota- 
lidad usando la historia en su favor (Hartman 2019). Otros países como Brunei, Filipinas y Malasia, también demandan una parte de las islas, argumentando que aquellas están dentro del rango de sus 200 millas marítimas de zona económica exclusiva determinados en la CONVEMAR (Arana 2015). Sin embargo, como se muestra en la figura, la RPC se ha apoderado de una gran parte del territorio marítimo que, legalmente, pertenece a Filipinas, Indonesia, Malasia y Vietnam.

La duda sobre el comprometimiento de la RPC con la CONVEMAR y la sospecha de expansionismo puede ser entendida desde diferentes aspectos. En 1992, el congreso de la RPC aprobó la "Ley sobre el Mar Territorial y la Zona Contigua de la República Popular de China", en la que reafirmaron la pertenencia de las Islas Spratly a la RPC (Fravel 2011, 294). Para complementar la sospecha de expansionismo de la RPC, los mapas 'oficiales' de aquel estado muestran que su territorio marítimo está delimitado por su línea de nueve puntos, la cual se puede observar también en la Figura 1 (Fravel 2011, 295). Esta línea ha sido base para la RPC. En 2011, la RPC hizo público a la Comisión de los Límites de la Plataforma Continental (CLCS) de la ONU que las islas son completamente parte de su mar territorial y su zona económica exclusiva (Fravel 2011, 295). Pero ¿qué hace a esta zona tan importante e interesante tanto para EE. UU., la RPC y los demás Estados involucrados en las disputas?

\subsection{Economía en la zona}

La importancia de la región Pacífica-Occidental para el balance de poder global puede explicarse desde diferentes enfoques dentro de la perspectiva económica. Uno de los enfoques consiste en la actividad comercial que ocurre dentro de esta zona. Según La Conferencia de las Naciones Unidas sobre Comercio y Desarrollo (UNCTAD), 80\% del comercio global se da a través de vías marítimas y, en la región Pacífica Occidental, el Mar del Sur de China acoge 1/3 del comercio marítimo global (China Power Project 2019). Esta área es vital para el comercio de las potencias mundiales, representando aproximadamente el $40 \%$ del comercio de la RPC y el 6\% de Estados Unidos (China Power Project 2019). Por estas razones, la región Pacífica-Occidental es una zona clave dentro de la economía global. 
Otra parte del análisis económico sobre la región Pacífica-Occidental tiene que ver con los recursos de potencial explotación que se encuentran en la zona. Según US Energy Information Agency, se conoce que el Mar del Sur de China contiene alrededor de 190 trillones de pies cúbicos de gas natural, más 11 billones de barriles de petróleo y, probablemente, existen 160 trillones de pies cúbicos de gas natural y 12 billones de barriles de petróleo todavía no identificados en la zona (Asia Maritime Transparency Initiative 2019). Estos recursos son explotados dentro de las zonas exclusivas económicas de China, Malasia, Vietnam, Filipinas, Brunei e Indonesia, como lo permite la CONVEMAR.

Estados Unidos, con completo conocimiento sobre la importancia de esta zona, mantiene distintas operaciones marítimas, con el fin de preservar un sistema basado en el respeto a las reglas del Derecho Internacional. La Séptima Flota de los Estados Unidos realiza operaciones de 'libertad de navegación' en el Mar del Sur de China y, a través de la Estrategia del Indo-Pacífico, ha intentado fortalecer alianzas dentro de la zona (Santoro 2019). Por el contrario, la política internacional de la RPC tiene un objetivo claro: la primicia política, económica y militar en la zona.

\section{Estrategias de la RPC para liderar en la región Pacífico Occidental}

\subsection{Estrategia política}

Una de las estrategias de la RPC ha sido establecer relaciones diplomáticas con Estados que han reconocido al gobierno de Taiwán como soberano para progresivamente aislarla (Santoro 2019). Aunque tales Estados tienen relativamente mínimo impacto dentro del Sistema Internacional, un aislamiento político y económico a Taiwán debilitará una posible respuesta de EE. UU. en defensa de Taiwán y de la región Pacífica Occidental, lo cual incluiría una fuerte pérdida en la influencia de EE. UU. en la zona (Santoro 2019). Desde 2016, Taiwán ha perdido el reconocimiento de siete Estados que lo reconocían como independiente, siendo Kiribati y las Islas Salomón los más recientes (Lyons 2019a). El fuerte cabildeo de la RPC sobre los países del Pacífico consiste en que la potencia ofrece productivas relaciones comerciales a cambio de cortar 
relaciones diplomáticas con Taiwán. Esta situación permanece causando la pérdida de poder de EE. UU. en la región del Pacífico Occidental (Lyons 2019b).

\subsection{Estrategia militar}

Durante los últimos 6 años, la RPC ha construido 7 nuevas islas sobre arrecifes en la zona de las Islas Spratly con el propósito de su la militarización (Asia Maritime Transparency Initiative 2017). Xi Jinping, presidente de la RPC, y su ministro del exterior han mencionado que la militarización de estas islas es necesaria para la seguridad marítima y brindar ayuda en caso de un desastre natural. Sin embargo, las bases militares, puertos navales y bunkers construidos por la RPC tienen realmente como fin guardar combustible, armas y misiles para la defensa aérea y marítima (Stashwick 2019).

Además, a través del uso de la estrategia denominada Cabbage strategy, estas islas militares le han servido a la RPC para controlar la zona y presionar a los demás países que reclaman territorio en ellas. Esta estrategia consiste en rodear un área de mar determinada, en este caso una isla, con la mayor cantidad de botes, marineros, barcos de vigilancia, y barcos de ataque que sean posibles, logrando así bloquear la entrada a dicha área (Kazianis 2013). Por lo tanto, como la RPC tiene sus nuevas bases militares en la zona disputada, puede ejercer un fuerte poder coercitivo a través de esta estrategia, ya que no ataca directamente a las islas de algún Estado o a sus habitantes. Es decir, no existe un proceso de coerción militar desde adentro de las Islas, lo que sería una intervención militar. Lo que logra la RPC a través de esta estrategia es presionar desde afuera, es decir, rodea tal territorio con diferentes tipos de navío y logra aislar la entrada de recursos a tal territorio por lo que, ante la eminente presión, la RPC termina adquiriendo el control de las islas, resignando al Estado que previamente la controlaba.

\section{5. ¿Existe similitud entre los hechos y el Realismo?}

Los esfuerzos políticos y militares de la RPC son signos de su deseo de invertir las dinámicas de poder en la región Pacífica Occidental a su 
favor. Los hechos previamente presentados pueden ser analizados desde la Teoría Realista de RR. II. En específico, el Realismo Ofensivo es útil por las similitudes que presenta el comportamiento de la RPC con tal modelo. Según esta teoría, cuando un Estado racional está creciendo con relación a otros Estados potentes, este percibe el poder como la única fuente de seguridad restante y, para asegurar su supervivencia en el Sistema Internacional, busca maximizar su expansión (Cox y Stokes 2012, 9-10).

Por tal razón, teóricamente y en base a los hechos previamente explicados, al ser la RPC un Estado creciente alrededor de potencias mundiales como EE. UU., está buscando maximizar su expansión dentro de la zona Pacífico Occidental y el Mar del Sur de China. Pues es un espacio sumamente importante en la economía mundial, y es su manera principal de adquirir poder, seguridad, y sobrevivir entre las demás potencias globales. Además, el Realismo Ofensivo expresa que la mejor manera de asegurar la sobrevivencia en el sistema es ser el Estado con más poder dentro de él y, aunque tener hegemonía global es prácticamente imposible en el sistema, la hegemonía regional es completamente posible para cumplir los enunciados de esta teoría (Cox y Stokes 2012, 10). Comparando nuevamente el Realismo Ofensivo con la situación de la RPC en la región Pacífico Occidental, es predecible que la RPC intente obtener y consolidar su influencia en su propia región para ser uno de los Estados con más poder dentro del sistema.

Adicionalmente, desde el Realismo Ofensivo se pueden explicar las crecientes tensiones que han surgido entre EE. UU. y la RPC. Según esta teoría, cuando un Estado adquiere hegemonía regional, este tiende a intentar prevenir que otros Estados realicen lo mismo dentro de sus regiones, con el fin de prevalecer como el Estado con más poder en el sistema (Cox and Stokes 2012, 10). Tal fenómeno genera tensiones entre los Estados. Por lo tanto, si relacionamos esta teoría con la hegemonía regional y el alcance prácticamente global que ha tenido EE. UU desde 1945, se entiende el origen de las tensiones en la región Pacífico Occidental que mantiene con la RPC. Por un lado, la política de EE. UU. con respecto a la región Pacífico Occidental radica en asegurar su posición, influencia 
y liderazgo en la región, mientras que la RPC ha establecido una política radicada en la expansión de influencia y alcance en la región y en el mundo. Esto puede desembocar en confrontaciones violentas entre EE. UU. y la RPC.

\section{Conclusión}

¿Existe la posibilidad de un giro en las dinámicas de poder en la región Pacífico Occidental? Después del análisis generado, podemos decir que efectivamente está creciendo la posibilidad de un cambio en las dinámicas de poder dentro de la región Pacífico Occidental. Si consideramos los hechos militares, económicos, políticos y sociales que se explicaron previamente en este artículo, es plausible decir que la República Popular China tiene una fuerte posición Realista Ofensiva en su estrategia nacional. Al basar la política de un Estado en el Realismo Ofensivo, según $\operatorname{Kim}(2015,113)$, no sería extraño que una potencia intente asegurar sus fronteras, redefinir los bordes de su territorio, buscar el acceso a nuevos mercados, recursos, rutas de transporte, entre otros aspectos.

Por lo tanto, la RPC ha utilizado, y utilizará en el futuro políticas basadas en el Realismo Ofensivo, con el fin de establecer control y su hegemonía en la región. Por todos estos motivos, en conclusión, creemos que efectivamente existe posibilidad para un cambio en las dinámicas de poder dentro de la región Pacífico Occidental, en la que el estatus quo de EE. UU. en la zona puede pasar a manos de la RPC. Sin embargo, no podemos concluir en que efectivamente va a haber un giro en las dinámicas de poder en la región Pacífico Occidental en la que la RPC gane el liderazgo completo y EE. UU. pierda su completamente influencia en la zona, ya que EE. UU. ha establecido en sus intereses principales defender su estatus en la zona. Como se mencionó previamente, EE. UU. ha construido su influencia en la zona a través de la creación de alianzas formales y políticas, y la promulgación del respeto al Derecho Internacional, con el fin de detener las aspiraciones de expansión de la RPC. 


\section{Referencias}

Allison, Graham. 2016. "Of Course China, Like All Great Powers, Will Ignore an International Legal Verdict." The Diplomat, July 11, 2016. https://learn.bu.edu/ bbcswebdav/pid-7198127-dt-content-rid-31552999_1/courses/19fallcasir373_al/ Allison2016.pdf.

American Institute in Taiwan. 2019. “Taiwan Relations Act (Public Law 96-8, 22 U.S.C. 3301 et seq.)." Recuperado noviembre 1, 2019. https://www.ait.org.tw/ our-relationship/policy-history/key-u-s-foreign-policy-documents-region/taiwan-relations-act/.

Arana, Ismael. 2015. “Las cinco claves de la disputa territorial por el mar de China Meridional." El Mundo, octubre 27, 2015. https://www.elmundo.es/internacional/2015/10/27/562f84e746163f59648b4689.html.

Asia Maritime Transparency Initiative. 2017. "A Constructive Year for Chinese Base Building." Center for Strategic and International Studies. Recuperado noviembre 1, 2019. https://amti.csis.org/constructive-year-chinese-building/.

Asia Maritime Transparency Initiative. 2019. "South China Sea Energy Exploration and Development." Center for Strategic and International Studies. Recuperado noviembre 1, 2019. https://amti.csis.org/south-china-sea-energy-exploration-and-development/.

Black, Ben. 2018. "The South China Sea Disputes: A clash of international law and historical claims." Journal of Law and International Affairs, marzo 22, 2018. https://sites.psu.edu/jlia/the-south-china-sea-disputes-a-clash-of-internationallaw-and-historical-claims/.

China Power Project. 2019. "How much trade transits the South China Sea?" Center for Strategic and International Studies. Recuperado noviembre 1, 2019. https://chinapower.csis.org/much-trade-transits-south-china-sea/.

Congressional Research Service. 2018. The Asia Pacific: Challenges and Opportunities for U.S. Policy, por Emma Chanlett-Avery, K. Alan Kronstadt, Susan V. Lawrence and Mark E. Manyin. Recuperado noviembre 1, 2019. https://fas.org/sgp/crs/ row/IFl1047.pdf.

Council on Foreign Relations. 2019. "U.S. Relations with China" Recuperado no- 
viembre 1, 2019. https://www.cfr.org/timeline/us-relations-china.

Cox, Michael y Doug Strokes. 2012. U.S. Foreign Policy (2nd edition). Oxford: Oxford University Press.

Department of State. 2019. “The Chinese Revolution of 1949”. Recuperado noviembre 1, 2019. https://history.state.gov/milestones/1945-1952/chinese-rev.

Desjardins, Jeff. (2019). “Chart: The World's Largest 10 Economies in 2030." Visual Capitalist, enero 11, 2019. https://www.visualcapitalist.com/worlds-largest-10-economies-2030/.

Fravel, Taylor. 2011. "China's Strategy in the South China Sea." Contemporary Southeast Asia: A Journal of International and Strategic Affairs 33 (3):292-319. 10.1353/ csa.2011.0136.

Hartman, Leigh. 2019. "The importance of the South China Sea." Share America, Julio 11, 2019. https://share.america.gov/importance-of-south-china-sea/.

Hook, Steven and John Spanier. 2017. American Foreign Policy Since World War II (20th Edition). Los Angeles: CQ Press.

Kazianis, Harry. 2013. “China's Expanding Cabbage Strategy." The Diplomat, octubre 29, 2013. https://thediplomat.com/2013/10/chinas-expanding-cabbage-strate$\mathrm{gy} /$.

Kim, Jihyun. "Territorial Disputes in the South China Sea: Implications for Security in Asia and Beyond." Strategic Studies Quarterly 9, no. 2 (2015): 107-41. http:// www.jstor.org/stable/26271078.

Lyons, Kate. 2019a. "China extends influence in Pacific as Solomon Islands break with Taiwan." The Guardian, septiembre 16, 2019. https://www.theguardian. com/world/2019/sep/16/china-extends-influence-in-pacific-as-solomon-islands-break-with-taiwan.

. 2019b. "Taiwan loses second ally in a week as Kiribati switches to China" The Guardian, septiembre 20, 2019. https://www.theguardian.com/world/2019/ sep/20/taiwan-loses-secsond-ally-in-a-week-as-kiribati-switches-to-china. 
Organización de las Naciones Unidas. 1982. "Convención de las Naciones Unidas sobre el Derecho del Mar". Recuperado noviembre 1, 2019. https:// www.un.org/Depts/los/convention_agreements/texts/unclos/convemar_ es.pdf.

Santoro, David. 2019. "Beijing's South China Sea Aggression Is a Warning to Taiwan." Foreign Policy, septiembre 16, 2019. https://foreignpolicy.com/2019/09/16/beijings-south-china-sea-aggression-is-a-warning-to-taiwan/.

Stashwick, Steven. 2019. "China's South China Sea Militarization Has Peaked." Foreign Policy, agosto 19, 2019. https://foreignpolicy.com/2019/08/19/chinas-south-china-sea-militarization-has-peaked/.

The White House. 2017. National Security Strategy of the United States of America. Por The White House. Washington DC. 